\title{
Conditions de l'élevage dans les llanos vénézuéliens
}

\author{
par R. FIASSON
}

\section{La terre (Géographie).}

Llanos. - « Mot employé dans la Nouvelle-Grenade et au Venezuela pour désigner de vastes plaines d'herbes hautes et abondantes ».

C'est ainsi que le Littré nous définit ces immenses pampas tropicales qui, au Venezuela, s'étendent sur une superficie dépassant le tiers du pays.

I.e Venezuela comprend $1.043 .000 \mathrm{~km}^{2}$ soit approximativement deux fois l'étendue de la France; sa population dépasse à peine 3 millions d'habitants.

Lorsqu'en 1493. Christophe Colomb découvrant la partie orientale de l'Amérique du Sud, s'engagea dans le golfe du Paria, ses matelots étonnés par la puissance du fleuve qui sortait de ce mystérieux continent et dont le courant ocre, chargé d'herbe et de limon, venait battre les côtes de Trinidad, lui demandèrent où il prenait sa source.

Christophe Colomb, sans hésitation, leur répondit : " Dans le Paradis Terrestre ».

Aussi, les conquistadores qui s'engagèrent sur ce chemin des eaux, croyaient-ils partir à la découverte du lieu merveilleux d'où leurs premiers ancêtres avaient été chassés par l'Archange, et nul insuccès ne pouvait les rebuter dans cette poursulte vers l'inconnu.

Un an plus tard, Alonso de Ojeda suivant la côte nord s'enfonçait dans le lac de Maracaỉbo. Des huttes misérables, bâties sur pilotis, étaient reliées à la rive par de fragiles passerelles de bois. Les compagnons de Ojeda parmi lesquels le Florentin Amerigo Vespucci, qui avait connu les splendeurs des palais de Venise, déçus et amers devant cette découverte si éloignée de leurs rêves, par dérision, baptisèrent ce nouveau pays : Venezuela (la petite Venise).

Un siècle derrière Christophe Colomb, l'Anglais Sir Walter Raleigh remontait aussi le cours de l'Orénoque. Il ne recherchait plus un paradis perdu, mais il avait encore les yeux fixés sur un horizon de mirages. Il s'attendait à chaque instant, sur l'immensité des llanos, à voir surgir devant ses yeux éblouis, la cité resplendissante, la vilie d'cr de Manoa, dent le prince, le Dorado, protégé par sa cuirasse de séquins d'or, promenait sa mélancolie tout le long d'un lac plein du même fabuleux métal.
Pas une légende indienne, pas une hallucination de soldat égaré, qui ne fit apparaître, aux yeux avides des conquérants, la ville des rêves.

De nos jours encore, dans l'immensité de ces territoires non explorés, dans l'État de Bolivar en particulier, dont les trois quarts restent à découvrir, la même espérance reste cachée au fond des pensées du chercheur d'or et de diamants qui poursuit ses recherches, souvent vaines, autour des villes sans routes, sans femmes, sans alcocl, sans armes, qui ont poussé sur la frontière du Brésil. C'est aussi l'espoir secret du voyageur qui vient se heurter aux flèches des Guahahibos du Haut-Orénoque.

Pour celui-ci, si les Indiens défendent l'entrée de ces territoires c'est qu'ils en ont reçu l'ordre de quelque roi puissant et lointain, désirant mettre ses richesses à l'abri de cupides regards.' Pour celui-là, la forêt cache des trésors, des villes ensevelies sous la jungle, où des singes-araignées jouent avec de monstrueuses émeraudes. Il ne s'agit que de les découvrir.

Aussi que de légendes, que de croyances, que de désespoirs et que d'épopées ridicules (des jeunes gens qui meurent de faim après avoir mangé leur chien !) ne provoquent pas le mirago do ces mots inscrits sur les cartes: " unsurveyed » (inexploré).

$\mathrm{Au}$ Venezuela ces territoires sont encore immenses, bien que moins étendus que dans le bassin de l'Amazone. Mais la pampa elle-même, plus facile cependant à parcourir, porte des taches inconnues. Ainsi tout le sud de l'État de l'Apure reste à découvrir. Nous avons pu, en 1946, relever un cours d'eau d'une vingtaine de mètres de largeur qui n'était signale jusqu'alors sur aucune carte.

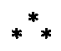

Llanos ! caractéristique essentielle du Venezuela et de la Colombie qui s'étendent depuis la chaine qui borde les côtes du Caracas jusqu'aux forêts de la Guyane, depuis les monts couverts de neige de Merida jusqu'au grand delta formé par l'embouchure de l'Orénoque.

L'altitude moyenne de ces steppes est comprise 
entre 50 et 300 mètres. Contrairement à ce que l'on pourrait supposer ces terrains ne constituent pas une plaine rigoureusement horizontale recouverte de graminées. On y trouve de léyères surélévations appelées «bancos de sabana » qui ne s'inondent pas en hivernage et constituent, par conséquent, des parties appréciées, et parfois âprement dispu'tées, par les éleveurs. D'autres parties, légèrement plus basses, forment les "esteros 》 ou 《'beijos'》 qui sont recouvertes d'eau pendant plusieurs mois de l'année. Lorsque les eaux se retirent, ces dépressions offrent un sol extrêmement bosselé, irrégulier sur lequel la marche est difficile. Gens et bêtes, cavaliers et conduteurs de véhicules, les évitent aussi soigneusement qu'ils le peuvent pour leurs déplacements. Quand un trajet conduisant d'un village au village voisin, d'une ferme à une autre, peut emprunter les' « bancos », c'est la voie qui sera choisie même si cela l'allonge considérablement.

Les llanos présentent aussi des parties boisées rassemblées le plus souvent en bordure des cours d'eau « rios » et parfois « canos » (le cano est un petit cours d'eau qui se créé à partir de sentiers sur lesquels l'eau exerce plus rapidement une action de ravinement). Si l'épaisseur du bois est suffisante, tout le long des grands affluents de l'Orénoque, par exemple, il prend le nom de galerie forestière (selva de galeria).

Dans d'autres régions des llanos, plus particulièrement dans le Guarico et l'Apure, les bosquets forment des taches isolées au milieu de la savane, à la manière de parcs. On les appelle alors des « matas ».

Si l'on survole les llanos on aperçoit les traces de nombreux anciens lits de rivières que l'on appelle des « madres viejas ». Ils forment des chapelets de mares isolées qui parfois, en saison des pluies, deviennent de véritables tronçons de cours d'eau. Les changements fréquents de direction des « rios" qui sont à l'origine de ces «madres viejas » sont provoqués par une très faible différence d'altitude sur la longueur de leurs parcours et par l'absence d'accidents de terrain. Ces anciens lits sont précieux parce qu'ils gardent souvent de l'eau en saison sèche et servent à l'abreuvement des troupeaux; d'autre part, la fertilité de leurs rives est beaucoup plus grande que les terrains avoisinants.

Parmi les arbres les plus remarquables qui composent les bosquets des llanos sont le « saman », Smanea saman, le " guasimo », Guazuma ulmifolia, le « caracaro», Enterolobium cyclocarpum, tous les Irois constituant des ressources fourragères appréciables, par leurs fruits, à la fin de la saison sèche.

Le « chigo », Campsiandra comosa, est aussi très recherché pour la nourriture des porcs à l'époque de la maturation des gousses. Les indiens Yaruro en consomment la farine (1). Malheureusement' cette espèce n'est abondante que dans le sud de l'Apure, sur les rives des cours d'eau.

Viennent ensuite l' « aceite », Copaifera officinalis, le «pilon », Andina inermis, plusieurs éspèces du genre Erythrina de la famille des Papilionacées, le "merecure », Couepia guianensis, le' "merei ", Anacardium occidentale, le « parapara », Sapindus saponaria, des anonnes sauvaiges dont le «manirote», Anonna purpurea, le " mata palo » Ficus sp. qui étouffe les arbres autour desquels il s'enroule; les goyaviers sauvages (especes de la famille des Myrtacées), le « mata raton », Glyricidia sepium, des «lecheros» (plusieurs espèces d'Euphorbiacées, le « dividive », Caesalpinia coriceria, le « jobo», Spondias lutea, le « ciruelo», Spondias purpuera, le « canafistolo », Cassia moschata, « indio desnudo », Bursera simaruba, le «tacamahaco», Protium heptaphyllum, le « algarrobo, Hymenaea courbaril, « mamon », Melicocca bijuga dont on mange les fruits. Le manguier, Mangifera indica, bien que d'origine étrangère s'est si complètement naturalisé qu'on le rencontre un peu partout.

Bien entendu des variations importantes dans cette flore arbustive se remarquent selon la partie des llanos qui est considérée. Car dans cette:zone il nous faut délimiter une partie élevée, en manière de corniche touchant à la montagne des Andes, et une autre, basse, formée par les savanes et les vallées au fond desquelles coulent les' grandes rivières. Ia ligne de séparation serait la courbe de niveau correspondant à 100 mètres d'altitude environ.'

Les parties hautes, souvent très arides; qui portent le nom de tables ( mesas ») sont parsemées d'un arbuste très résistant à la sécheresse et à l'incendie; au tronc recouvert d'une écorce épaisse: cassant comme du verre, le « chaparral » Byrsonina crassifolia.

Là où il pousse l'eau est rare et les perforations si profondes soient-elles ne trouvent pas toujours la nappe phréatique.

Par contre dans les parties l'es plus basses et les plus humides des llanos se dresse le magnifique palmier « morichal » Mauritia flexuosa qui compose des palmeraies plus ou moịns étendues.

Le cavalier les évite avec soin car ce palmier a très souvent le pied enfoncé dans un marécage d'où il est difficile de sortir. Mais débouchant de la fondrière un ruisselet d'eau claire serpente à travers la savane pour la grande joie des hommes et des

(1) Analyse chimique d'un échantillon de cette farine :

Humidité...... 15,51 \% Fibre crue ... . 1,31\% Extrait éthéré. $\quad 0,37 \%$ Cendres..... $0,71 \%$ Protéine .... 6,68\% Hydrate de C . $75,38 \%$ 
animaux assoiffés par le soleil implacable et le vent desséchant.

Le palmier " morichal 》 est la providence de ces étendues désolées; tout.en est utilisé par l'habitant de la pampa. Le tronc et ses feuilles pour sa demeure, ses fibres pour tresser ses hamacs, sa sève comme vin et son cœur comme farine.

Dans les llanos plus secs un autre palmier qui n'a pas l'élégance du "morichal 》 rend aussi de précieux services. C'est la « palma llanera », Copernicia tectorum. Il est souvent accompagné de l'arbre classification. Sa superficie atteint $45 \%$ du territaire vénézuélien et la densité de son bétail est importante tout au moins dans sa partie Nord-orientale.

Apure, Barinas et Portuguesa sont les trois États des llanos occidentaux.

La région du Haut-Apure, au sud de Barinas et le sud de Monagas sont certainement les zones d'ćlevage d'un grand avenir à cause de la fertilité de leurs terres alluvionnaires, de leur richesse en eau, de leur climat moins pénible et de leur topographie beaucoup plus plate que partout ailleurs.

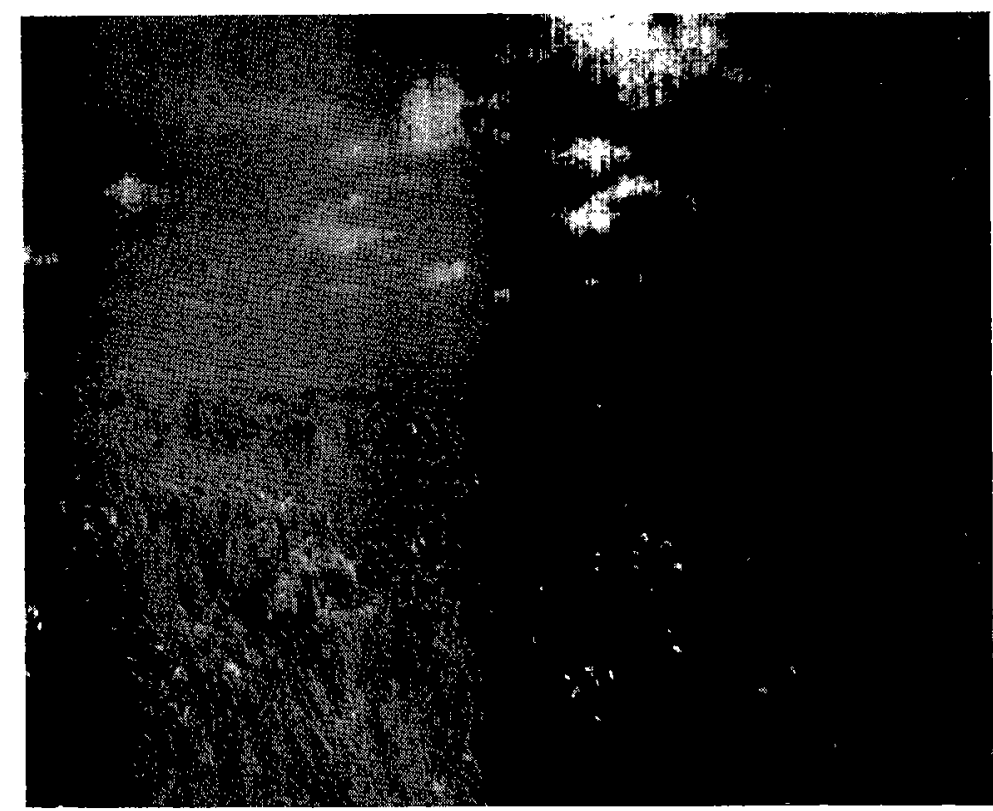

Fig. 1. - Les llanos.

(Cliché Mme Fiasson).

national de Venezuela, l' « araguaney », Thecoma chrysantha.

Bien qu'à ce sujet les opinions soient partagées il semble bien que les llanos, qui se sont sans doute formés par apport d'alluvions, ont été amplement boisés tout au moins dans les parties méridonale et occidentale.

Géographiquement nous pouvons distinguer les llanos centraux, les llanos occidentaux et les llanos orientaux.

Les premiers comprennent les États de Cojedes et Guarico. Les llanos orientaux : Anzoategui et Monagas. L'État du Bolivar (Guyane vénézuélienne) n'est pas considéré comme llano car son relief est très accidenté, mais nous le ferons entrer dans cette
Actuellement c'est d'ailleurs de l'Apure et du Guarico d'où provient la plus grande partie des boufs "novillos " qui sont amenés aux abattoirs centraux.

C'est dans les llanos de l'Apure que la plaine apparaît, parfois immense et nue, jusqu'à l'horizon. La steppe est infinie, elle semble s'élever autour du voyageur comme l'océan autour du navire.

La géographie de l'élevage au Venezuela nous indiquerait d'autres zones comme le bassin du lac de Maracaibo, l'État do Zulia, la cordillère des Andes (Merida, Trujillo, Tachira) et la zone d'embouche aux environs de Maracay et de Valencia dans les riches États de Aragua et de Carabobo. Mais l'apport de ces régions, s'il est important de par la valeur des animaux qui y sont élevés, est en vérité infime en nombre dans la masse des grands troupeaux vénézuéliens. 
Aux environs du lac Maracaỉbo, des éleveurs avisés ont crée des prairies artificielles gagnées sur la forêt; il's' y entretiennent à peu près convenablement des vaches laitières. Le lait ramassé par bateau, par train, par camions et même par avions, alimentait l'usine de Santa Barbara de Zulia qui fabriquait du lait concentré sucré et du lait en poudre.

Il semble d'ailleurs que cette usine ait cessé ses activités à cause du prix de revient trop élevé de ses fabrications.

La zone des Andes ne produit que quelques vaches laitières et quelques chevaux.

Enfin, les pâturages d'embouche aux environs du lac Valencia finissent seulement des animaux qui en grande majorité sont amenés des llanos. On y élevait aussi des races laitières mals dans des conditions souvent si peu avisées, en particulier en ce qui concerne la lutte contre les parasites internes ou leur prévention, que des vaches de race introduites à grands frais, suiccombent souvent avant même d'avoir rendu quelques services. Nous avons assisté ainsi à des hécatombes d'animaux de race Friesland décimés par Hemonchus contortus.

Selon le recensement de 1929 (" Iies cuirs exotiques, et le marché d'Anvers $\%$ ), le bétail vénézuélien pouvait être dénombré à 3.500 .000 têtes; les chiffres pouvant varier de 2.700 .000 à 3.500 .000 selon les diverses estimations. "Le bétail, précise l'auteur, vit dans la proportion de $85 \%$ dans les llanos, immenses plaines arrosées par les affluents andins de l'Orénoque. i Le seul État de l'Apure accusait près de 700.000 animaux dans ce recensement de 1929; celui de Guarico passait les 400.000 .

Qu'on l'admette parfois avec réticence, c'est bien explicable car. la réputation d'un pays d'élevage ne se fait pas avec des hordes de troupeaux vivant à l'état sauvage, exploités selon les procédés les plus primitifs et souvent les plus irrationnels. Il n'en est pas moins vrai que l'élevage bovin du Venezuela est à peu près réservé aux régions des llanos. Les troupeaux que l'on pourrait y dénombrer avec des recensements jamais faits et d'ailleurs impossibles à faire (le propriétaire ignorant lui-même souvent à des milliers près le nombre d'animaux qui vivent sur ses pampas) formeraient sans doute les quatre cinquièmes du cheptel total du Venezuela qui pourrait être estimé actuellement à 3.000 .000 de têtes environ.

Tíoubli de la zone des llanos est surtout d'ordre international. Lors des conférences qui rassemblent des techniciens de différents pays, comme à la réunion 1952 de l'Office Intennational des Épizuoties, il est aisé de convaincre les interlocuteurs de la disparition de la fière aphteuse quand on passe sous silence la zone des llanos.

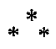

Les pâturages naturels de ces plaines splendides sont constitués par une grande variété de plantes fourragères parmi lesquelles les graminées prédominent.

La plus appréciée est certainement la « lambedora », Leersia hexandra, qui affectionne les parties basses (Esteros) et les bordures des lagunes surtout dans le Haut-Apure, la " bermuda », connue encore sous le nom de «pelo de indio », le chierident, Cynodon dactylon (que les américains nomment, aussi Bermuda grass), le «tucupen » ou « jeguey », Sporobolus indicus, dont une espèce voisine, Sporobolus virginicus, peuple les terrains de parcours des indiens de la Goajira situés près de la meri, le " Gamelotillo », Paspalum plicatulum et dans le même genre la Gamelote d'Apure, Paspalum fasciculatum, vers Anzoategui, Paspalum coryphaeum ainsi que plusieurs autres comme $P$. notatum, $P$. conjugatum, $P$. repens, dans les lieux humides de l'Apure et du Guarico, $P$. fimbriatum "paja de conejo ", très envahissante. Les pâturages de Zulia et Aragua sont aussi composés d'un Paspalum appelé " paja cabezona,", $P$. virgatum. La « paja carretera » Eragrostis maypurensis, est très fréquente sur les « bancos », en particulier sur les bords des sentiers.

La k Barbacoa », Axonopus compressus, assez fréquente dans les savanes fraîches qui échappent aux incendies.

Parmi les Panicum, en dehors des espècos importées, les llanos sont peuplés de Panicum laxum (paja de raiz) dans les parties basses et humides et Panicum fasciculatum (Granadilla) qui pousse surtout dans les terres riches qui ont été précédemment cultivées.

Sur les parties plus sèches, sur les bancos qui ne s'inondent pas, des Andropogon: A. bicornis (paja de zorro), A. selbanus (paja de sabana).

$\mathrm{Ce}$ dernier nom vernaculaire englobe anssi une espèce d'Aristida, A. adscensionis, que l'on trouve sur les llanos secs et élevés, sur les "mesas » et qui constitue, avec la "paja peluda », Trachypogon plumosus, et la "saeta 》, Trachypogon montufari, les plus mauvais fourrages poussant dans la zone des llanos.

Signalons enfin quelques espèces moins communes comme Antiphora hermaphrodita, Eleusina indica, Chloris mollis et C. polydactyla, Luziola pittieri (dans les lieux marécageux), Digitaria horizontalis, Eriochloa punctata, Pennisetum setosum, Imperata cylindrica, connue sous le nom de "vibora,». (vipère).

Dans les parties orientales de l'État d'Apure (Bas-Apure) qui sont inondées durant tout l'hivernage et qui constituent des pâturages d'embouche pendant la saison seche, on trouve une espèce du genre Echinochloa. 
Heteropogon contortus (graminée qui forme la base de pâturages très étendus à Madagascar où elle est connue sous le nom de "danga »), a été signalée dans plusieurs régions du Venezuzla (Pittier).

Ceci n'est pas pour nous surprendre.

La houlque de Bretagne, Holcus lanatus, a été rencontrée par Tamayo dans l'État de Aragua, sur le bord des chemins, et nous avons pu la recueillir dans l'île de la Nouvelle-Aimsterdam (Iles françaises australes). La Melinis minutiflora (capim gordura des brésiliens), originaire d'Afrique, est cultivée au Venezuela et indigène, ou naturalisée, à Madagascar, sous le nom de "menakapaha".

La " Gamelote ", Panicum maximum, semble bien indigène dans certaines régions de l'Apure et cette variété ne se distingue de l'herbé de Guinée que par une dureté plus grande de la tige.

Quelques légumineuses y ont aussi leur place. Les plus répandues appartiennent au genre Desmodium : D. purpureum (pega pega), D. canum (pata de danta), $D$. molle (bojoro); d'autres se rattachent au genre Vigna : $V$. vexillata et $V$. luteola (Bejuco marullero).

Il existe enfin des légumineuses appelées « frijollilo " qui font partie des genres Teramnus (une espèce existe aussi dans les pâturages de l'Ouest malgache), Controsoma et Calopogonium.

Parmi d'autres plantes indigènes qui, dans les llanos, méritent une mention spéciale par les services qu'elles rendent signalons le " dividive », Caesalpinia coriaria, dont on utilise les gousses, dans toutes les fermes, pour tanner les lassos. On laisse macérer les fruits dans l'eau durant deux jours puis on met la lanière de cuir à tremper pendant vingt-quatre heures dans la décoction.

Le "Caracaro », Enterolobium cyclocarpum, que nous avons déjà cité à cause de son importance dans l'alimentation des animaux, est utilisé par les artisans des llanos qui fabriquent des selles. L'écorce contient un tanin qui donne au cuir une belle couleur jaune paille tout aussi peu résistante à l'action du soleil que la couleur rosée donnée par le "dividive».

(La chaux est employée pour l'épilage des peaux).

C'est le tronc de ce caracaro que les llaneros utilisent pour la fabrication de leurs pirogues. C'est un bois assez léger, tout en étant très dur et très résistant, et à peu près imputrescible dans l'eau.

Dans la plaine, autour des villages, poussent en abondance des malvacées appelées "escobas", Sida rhombifolia, à grosses fleurs jaune pâle. Elles constituent une redoutable mauvaise herbe difficile à détruire, mais sont utilisées parfois comme plantes textiles.

La " curagua ", Bromelia sp., est commune aussi dans les llanos de l'Apure. On en tire un fil plus fin, utilisé pour des travaux plus délicats.

Une autre plante qui envahit avec facilité les terrains fertiles des savanes est le "mastranto ", Hyptis suaveolens, Les tiges peuvent atteindre 2 mètres de hauteur; les fleurs sont petites, bleues, et forment des épis. Cette plante aromatique, qui dégage une forte odeur de camphre, est utilisée dans la médecine populaire sous forme de bains contre les paralysies.

Cette plante se multiplie trop rapidement autour des fermes et diminue la valeur des pâturages mais elle est un signe précieux de la fertilité des terrains.

Des plantes toxiques se rencontrent soit dans les pâturages eux-mêmes soit dans les bosquets qui en rompent la monotonie. Parmi celles-ci, les plus remarquables sont les " Guachamaca ». Les habitants de l'Apure en signalent de deux espèces : le «Guachamaca blanco " et le "Guachamaca negro o morado ".

"Guachamaca blanco". - Les spécimens que nous avions étudiés dans la région de Mantecal (HautApure) sont de ce type Anacampta angulata Myers. ( $C$ 'est le botaniste Lasser qui nous a donne ce nom scientifique. Pittier le nomme Malouetia nitıda Spruce).

C'est un arbuste de deux à trois metres de hauteur dont la sève est laiteuse comme celle des euphorbes. Les fleurs sont petites, blanches, en grappes. Les fruits sont de petites baies rouges, mûres en septembre. Cet arbuste pousse dans les galeries forestières. Les indigènes racontent que le dindon sauvage (pahoui) mange ces baies et qu'à cette époque, la consommation de sa chair peut provoquer des nausées, des vertiges, des vomissements et même des empoisonnements entrainant la mort. D'autres disent que seule la moelle osseuse est dangereuse et qu'il faut soigneusement éviter de jeter les os aux chiens.

Nous avions présenté des feuilles de ce " guachamaca " à des animaux domestiques. Les vaches l'ont dédaigne mais les porcs en auraient fait leur nourriture si nous ne les avions empêchés.

Des éleveurs racontent que parfois les bovins mangent les feuilles de cette plante. Ceci pourrait peut-être, en effet, se produire à la fin de la saison sèche quand les pâturages sont maigres. Ils ajoutent qu'ils n'en meurent pas à la condition de ne pas les obliger à se déplacer. Cette observation, si elle se révélait fondée présenterait une singulière importance dans l'étude de la "borrachera", maladie qui sévit sur les bovins paturant sur la rive gauche de l'Orénoque avant l'arrivée des pluies. Les symptômes de cette curieuse maladie sont ceux d'une intoxication.

Il est possible aussi que les éleveurs aient 
conlondu une autre plante avec le « guachamaca»,

" Guachamaca morado ". - Cet arbuste a des fleurs pâles. La sève n'est pas laiteuse. Il provoquerait de violents maux de tête aux personnes qui se reposent à son ombre.

Dans les pâturages on trouve la « Borrachita »: Passiflora sp. Ce premier spécimen a été rapporté du. Sud d'Elorza (Haut-Apure). La racine est un tubercule. La tige est une plante grimpante. Nous n'avions vu ni les fleurs ni les fruits.

Elle est très commune sur les bords de l'Arauca. sous l'influence de la lumicre solaire: On sait d'autre part que la fin de la saison sèche, et le début de la saison des pluies, sont particulièrement dangereux parce que la rareté d'une nourriture 'convenable oblige les animaux à consommer des plantes toxiques qu'ils dólaissent lorsqu'ils ont. de bons pâturages, et aussi que certaines plantes poussent plus rapidement aux premières pluies et que les troupeaux en mangent do plus grandes quantitós.

"Borrachita n*2. " Araceae sp. est une petite plante dont la racine est aussi un tubercule. Nous l'avions

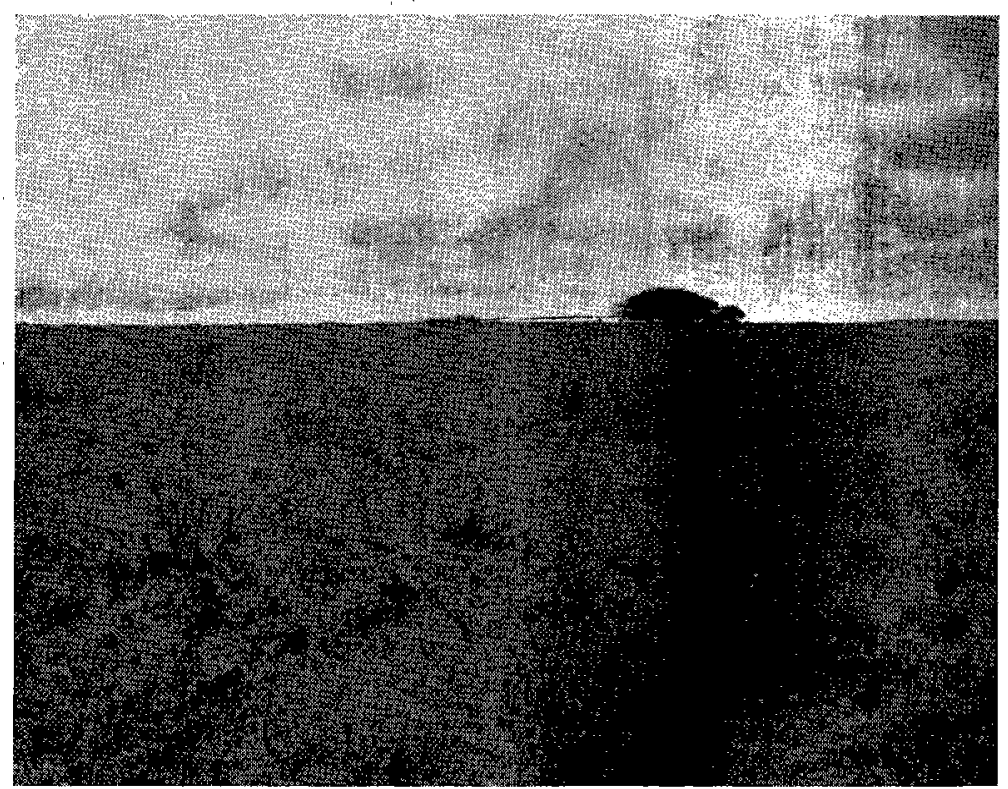

(Cliché Mme Fiasson).

Fig. 2. - Envahissement par les épineux.

Elle est redoutée des éleveurs de cochons car elle provoque une mortalité élevée sur ces animaux qui en mangent la racine. La mort survient en cinq ou six jours après le repas toxique. La graisse et la chair ont pris une couleur jaune très prononcée. Quelques personnes qui ont abattu les animaux avant la fin et qui ont mangé la viande n'en ont pas été incommodées.

Selon d'autres indigènes les bovins s'intoxiqueraient aussi en consommant les feuilles. Ils deviendraient alors comme ivres. Si on les laisse au repos les symptômes rétrocèdent : par contre, si on les oklige à marcher rapidement sous un soleil ardent, ils titubent, tombent et meurent. Si ces observations sont exactes elles concorderaient avec ce que nous savons d'autres plantes toxiques. Certains principes vénéneux ne peuvent agir, après absorption, que rapportée de la rive droite de l'Arauca, en face de l'hato Trinidad (Haut-Apure) où elle est très commune.

Les symptômes d'empoisonnement décrits par les éleveurs sont les mêmes que ceux provoqués par la passiflore d'Elorza.

" Barbasco. " - Tous les Indiens Yaruros en cultivent. On le trouverait aussi à l'état sauvage au Sud-Ouest d'Elorza. Les Indiens l'utilisent pour la pêche.

Curare. - Les Indiens qui vivent sur le Capanaparo (Yaruro) du Cinaruco (Yaruro, Guahibo et Chirikoa), du Juriepe (Guliguli - Cuiba ?) et du Meta (Guahibo) 'n'utilisent pas le curare, du moins pas à notre connaissance. Ceux que nous avions interrogés ignorent si l'on peut trouver des.Strychnos dans cette région. 


\section{$*^{* *}$}

En dehors des plantes cultivées que nous étudierons plus loin, nous avons encore rencontré dans certaines parties de l'État de Guyane le " toddy » Stizolobium, légumineuse sauvage de grande taille dont les gros haricots blancs sont consommés grillés, réduits en farine et délayés dans du lait.

Enfin, nous citerons la " chocha », Mucuna sp. dont nous n'avons vu qu'un exemplaire dans les llanos du Sud de l'Arauca (Haut-Apure) et nous

\section{$*^{*} *$}

Dans de nombreuses régions des llanos, sur la rive droite du rio Apure en particulier, entre les villages de San Vincente et Bruzual, il s'est développé en quelques années une véritable mer de buissons qui dépassent $1 \mathrm{~m} .50$ de hauteur. Ayant eu à nous déplacer à cheval nous avons perdu toute une journée à chercher un passage à travers ces mimosees qui s'étendent sur plusieurs dizaines de kilomètres et qui ont transformé cette partie de la pampa,

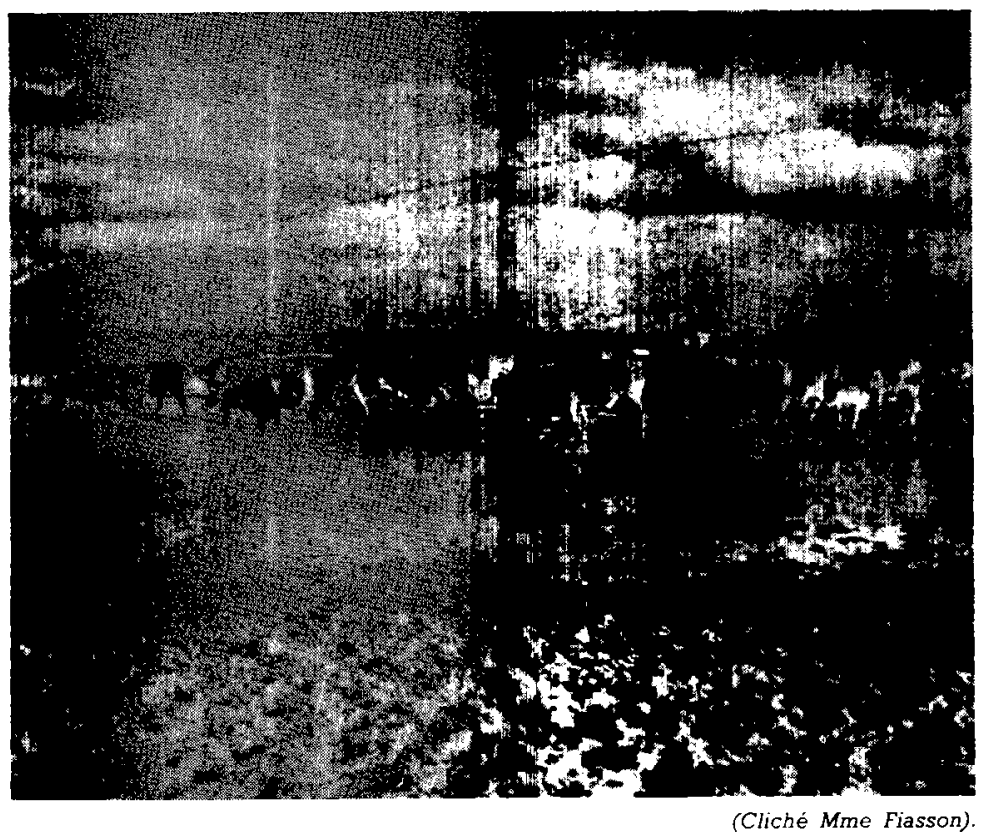

Fig. 3. - Troupeau dans une lagune.

ignorons si c'est une plante indigène et si elle se rencontre à l'état sauvage. C'est une légumineuse grimpante dont les fleurs, en grappe, sont d'une couleur de violette pâle. Les graínes sont des fèves grosses et irrégulières contenues dans d'énormes gousses, dures, larges et plates. On en tirerait une farine plus délicate que celle du riz. Cette plante donnerait des fruits en grande abondance de janvier à septembre et serait susceptible de constituer un apport précieux dans l'alimentation humaine et animale.

Elle pousserait très rapidement. Une bouture pourrait porter des fruits au bout d'un an.

Iues Guahibos font une énorme consommation de « yopo » ou « niopo » Piptadenia peregrina Benth: Ils prisent une poudre faite de graines grillées, ce qui leur procure un énivrement passag̀er. couverte antérieurement de pâturages, en un inextricable fourré où les animaux eux-mêmes ne pénètrent plus. Cet envahissement s'est produit en quelques années car en 1940, il y en avait fort peu. Et déjà entre les rios Guaritico et Setenta, affluents de la rive droite de l'Apure, dans une savane très dénudée où il n'y a même plus de bosquets (ni à plus forte raison de galeries forestières) ces buissons apparaissent de plus en plus disséminés çà et là, avec encore de larges passages entre les touffes. Dans cette région les pâturages ne sont pas chargés en animaux et cette végétation buissonneuse doit être la réaction du sol aux incendies périodiques des saisons sèches. Ce serait, en somme, à notre avis, un phénomène de défense de la nature pour la protection du sol.

- Par contre, plus en aval, vers les confluents de 
l'Apure et de l'Arauca avec l'Orénoque, dans les immenses savanes de l' " hato " national la Candelaria, qui sont surstockées en animaux et surtout en chevaux, des dunes sont apparues et l'apparence est nettement prédésertique. En 1947, en survolant ces savanes en avion, nous avions compté approximativement de 12.000 à 14.000 chevaux. En 1949, par contre nous n'en avons plus vu que quelques centaines sans qu'aucune explication de leur disparition puisse nous satisfaire.

La présence d'un aussi grand nombre de chevaux ou moins tardivement selon les années) se termine vers octobre. Les pluies les plus fortes sont en juillet et août. Les chutes d'eau s'étagent annuellement de $1 \mathrm{~m}$. 50 à $2 \mathrm{~m}$. 50. L'Orénoque et ses affluents débordent et inondent toutes les parties basses. du pays.

C'est alors la saison de l'abondance.

Des boufs gras, semi-aquatiques, enfoncés' dans l'eau jusqu'au cou, paissent des graminées juteuses et la pullulation extraordinaire des insectes ne peut rien sur l'organisme de ces animaux suralimentés.

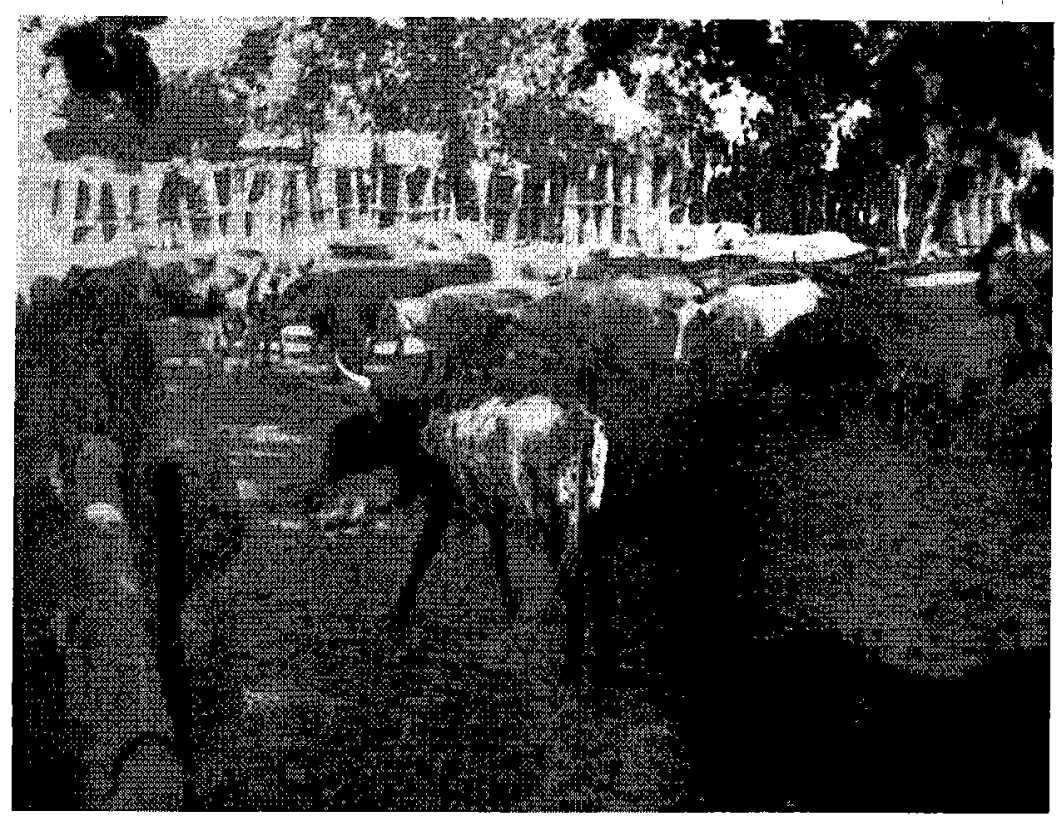

(Cliché Mmo Fiasson).

Fig. 4. - Boufs dans le corral (Halo LI Trio).

au milieu de quelque 20 à 25.000 bovins a eu certainement une influence désastreuse sur la végétation herbacée car leur façon de brouter détruit beaucoup plus le pâturage.

Climatologie. - Comprise approximativement entre le $8^{\circ}$ et $11^{\circ}$ degrés de latitude Nord, la région des llanos jouit d'une climat tropical.

La température moyenne est de $28^{\circ}$ à $30^{\circ}$ centigrades et en saison chaude on peut enregistrer des maxima de $40^{\circ}$.

Dans les llanos, donc, le soleil est lourd et la chaleur forte; mais ils sont supportables grâce à la brise qui souffle en permanence du nord-ouest, depuis 6 heures du matin jusqu'à 6 heures du soir. Les nuits sont généralement fraîches.

La saison des pluies qui commence en mai (plus
Les parties hautes et sablonneuses (les «bancos ») offrent alors un refuge au betail. C'est sur ces parties surélevées que lès animaux se rassemblent pendant la nuit. Dans certaines zones leur surface est parfois si limitée qu"on les appelle les "dormitorios de ganado " car ils ne peuvent constituer qu'un lieu de repos nocturne et non des pâturages d'hivernage.

Parfois, comme des îles dans l'océan, dans cette énorme surface inondée, des parties hautes servent de refuge permanent à des troupeaux de chevaux et de bovins qui deviennent très rapidement marrons.

Pendant cette saison appelée "invierno" (hiver) de profondes et étroites dépressions appelćcs " caños 》 qui réunissent souvent deux rios (et qui parfois s'allongent sur plusieurs kilomètres) se remplissent d'eau et convenablement endigués, peuvent 
constituer de remarquables réserves d'eau pour la saison sèche.

Cette zone inondable des llanos comprend tout le district de San-Fernando de Apure et une partie importante de celui de Achaguas dans l'État d'Apure, tout le Sud du district Miranda dans l'État du Guarico et la presque totalité du district Arismendi dans l'État de Barinas.

Dans ces régions et pendant cette saison, les déplacements s'effectuent en pirogue. Les montures s'épuisent trop vite dans les terrains inondés, et les "bancos " ne permettent pas toujours de se rendre à pied sec d'un point à un autre: Enfin les traversées des rios à la nage constituent de trop redoutables dangers pour que le llanero, pourtant normalement brave, les affronte d'une manière répétée.

Aussi, certains voyages en saison des pluies sont du domaine de l'impossible. Par exemple dans l'État de l'Apure les cours d'eau, affluents de l'Orénoque, coulant de l'Ouest vers l'Est, tout déplacement selon un méridien est une expédition longue et dangereuse, alors que dans le sens d'un parallèle, les chemins d'eau y constituent des moyens commodes.

Et les habitants des villages, perdus dans les llanos, nettoient avec soin les caños ou les rios qui constituent leurs uniques voies de communication.

Ainsi durant plusieurs mois les hommes poussent leurs embarcations sous des ciels d'orage et naviguent à quelques mètres au-dessus des os blanchis des animaux morts de soif quelques mois auparavant.
Les champs d'atterrissage eux-mèmes disparaissent sous les eaux comme c'est freoriemment le cas a San-Fernando de Apure et les rabitants reprennent leurs anciennes coutumes de vire séparés du reste du monde et de n'entretenir que des relations lentes et lointaines par la voie des illeuves.

Ces savanes restent inondées durant quatre à cinq mois ne surgissant de l'eau que les "bancos " et les « médanos », ces derniers constituant de petites collines qui, dans la plupart des cas. sont des emplacements d'anciens villages indiens de l'epoque précolombienne.

Lorsque la saison sèche fait son apparition, vers novenbre, les eaux se retirent et sur les terres inondees, pousse une riche végétation de graminées oi prédominent Leersia hexandra et Eragrostis maypurensis.

Le "verano» (été) (ainsi que les habitants des llanos nomment la saison sèche) est parfois d'une riqueur exceptionnelle. Dans les États du Guarico, Anzoategui, Portuguesa et Cojedes, le retard dans l'apparition des pluies peut causer des désastres, comme ce fut le cas en 1940 et en 1947, où un hivernage faible ne commença à remplir les "esteros" qu'en juillet.

Durant tout le "verano » les llaneros circulent à cheval. Seuls les plus pauvres des habitanis de la plaine marchent à pied. Le " peon " (le domestique) de la ferme, a toujours la monture qu'il utilise dans ses travaux de "rodeo". Camions et Jeeps cnt aussi envahi les llanos et circulent au milieu des troupeaux en soulevant des trombes de poussière. 